\title{
Factors Affecting Squatting Ability in Total Knee Arthroplasty Using High Flexion Prosthesis
}

\author{
Tiejian $\mathrm{Li}^{1,2}$ \\ Jingyang Sun ${ }^{1,2}$ \\ Yinqiao Du (iD) 2,3 \\ Zhisen Gao (D) ${ }^{2,3}$ \\ Haiyang $\mathrm{Ma}^{2,3}$ \\ Yonggang Zhou $\mathbb{D}^{1-3}$ \\ 'Medical School of Chinese PLA, Beijing, \\ 100853, People's Republic of China; \\ ${ }^{2}$ Department of Orthopedics, The First \\ Medical Center of PLA General Hospital, \\ Beijing, 100048, People's Republic of \\ China; ${ }^{3}$ Senior Department of \\ Orthopedics, The Fourth Medical Center \\ of PLA General Hospital, National \\ Clinical Research Center for \\ Orthopedics, Sports Medicine \& \\ Rehabilitation, Beijing, I00853, People's \\ Republic of China
}

Correspondence: Yonggang Zhou Department of Orthopedics, PLA General Hospital, Fuxing Road, Haidian District, Beijing, People's Republic of China

Tel +861380I287599

Email ygzhou30I@I63.com
Purpose: Total knee arthroplasty (TKA) is widely used as a treatment for knee osteoarthritis. Few studies have analysed the factors affecting the squatting ability of patients after TKA. The purpose of this study was to comprehensively analyse the factors affecting squatting ability after TKA and to determine which ones are important.

Patients and Methods: Three hundred primary TKA cases with a minimum 3-year followup were retrospectively analysed. All patients received a conventional posterior-stabilized TKA implant and underwent a standard perioperative care pathway. The patients were divided into two groups according to the squatting position and knee flexion angle while weight-bearing (Group I - inability to squat group, Group II - ability to squat group). Demographic, operative, and clinical data were collected. Radiographic assessment included joint line elevation, patellar position, posterior condylar offset (PCO), etc. Statistical analysis of the effect of all the above factors on squatting ability was performed.

Results: The preoperative range of motion and joint line of Group I were 82.9 \pm 12.6 and $3.24 \pm 1.07$, respectively, and those of Group II were $107 \pm 9.6$ and $1.83 \pm 0.89$ respectively. The univariate analysis showed that age, prosthesis size, preoperative ROM and joint line position were correlated with squatting ability. But in the final multivariate analysis, joint line position and preoperative ROM were independent influencing factors that affected squatting ability after TKA ( $\mathrm{p}$ value $<0.01$ ).

Conclusion: Preoperative ROM and joint line position were independent influencing factors affecting squatting ability after TKA. Patients should be counseled accordingly and be made to understand these factors. To ensure that patients can squat postoperatively, we should improve surgical techniques to control joint line elevation.

Keywords: squatting ability, joint line elevation, preoperative ROM, total knee arthroplasty

\section{Introduction}

Total knee arthroplasty (TKA) is an effective surgical intervention for patients with end-stage knee joint degeneration. ${ }^{1}$ The primary goals of TKA are to relieve pain and improve knee function, thereby improving the patient's quality of life. Satisfaction rates after TKA range from 75 to $90 \%{ }^{2}$ Squatting, which covers a wider range of knee flexion than gait, is one of the most unsatisfactory motions after TKA. ${ }^{3}$ Especially for Asian people, squatting is very important in daily life, because there are many movements in Asian lifestyle that require certain squatting ability to complete. ${ }^{4}$ Many daily activities require knee flexion greater than $90^{\circ}$, whereas squats require knee flexion of $110^{\circ}$ to $130^{\circ} .5,6$ The classification of squat positions is commonly seen in studies of weightlifters, which are usually divided into half squats (knee flexion $80-100^{\circ}$ ), horizontal squats $\left(110-120^{\circ}\right.$ ) and deep 


\section{Graphical Abstract}

\section{Factors affecting squatting ability in total knee arthroplasty}

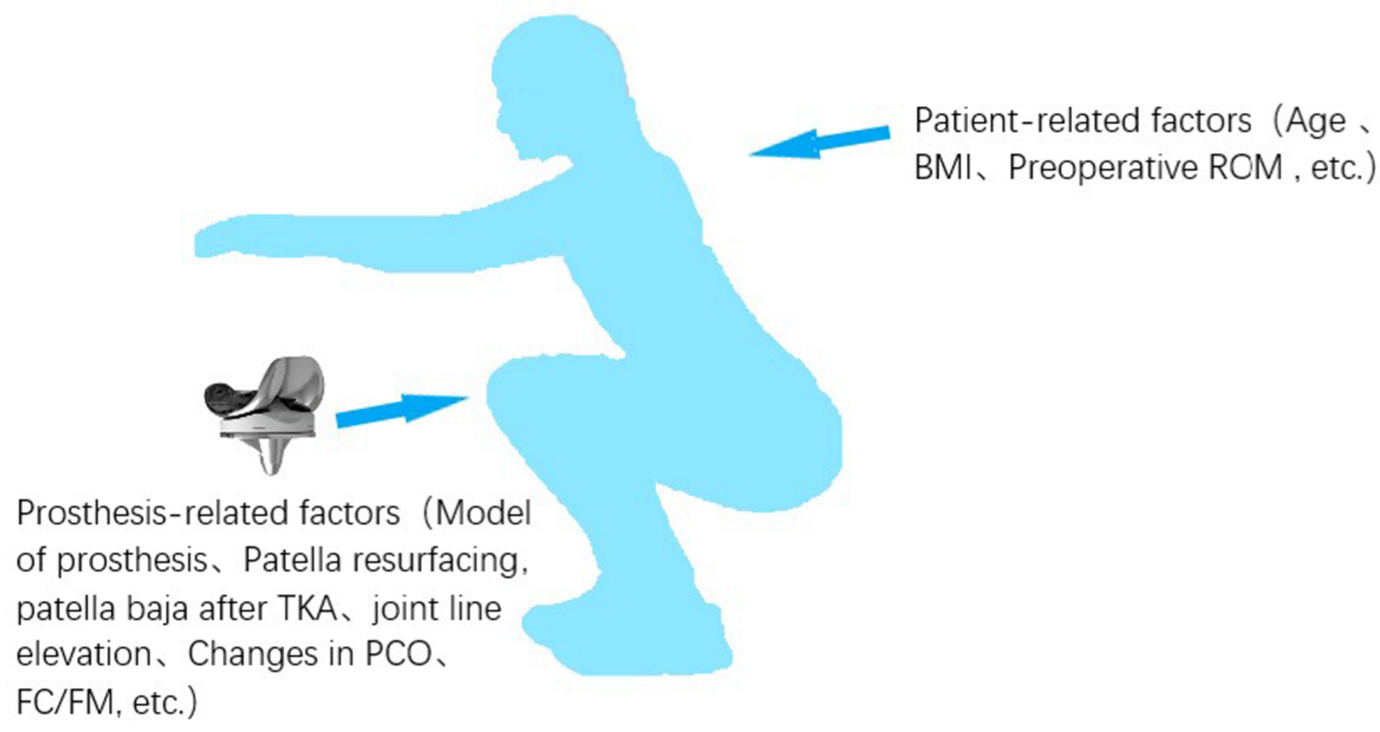

squats (above $135^{\circ}$ ) according to the depth of the squat. ${ }^{7}$ Deep squat requires the knee to flex at least $135^{\circ}$. For Asian people, many movements in daily life require deep squats, such as the Asian squat, the use of squatting stool and kneeling for religious beliefs. Therefore, deep squatting is the standard to determine whether patients have the ability to squat after TKA.

At present, many studies have focused on factors affecting the range of motion (ROM) of patients after TKA, whereas few studies have examined the influence of patients' postoperative squatting ability. ROM after TKA depends on the individual patient, joint line (JL), patella baja, implant, and many other factors. ${ }^{8-10}$ Individual patient factors have included BMI, age, and preoperative ROM. Studies on the effect of JLs on postoperative mobility are the most reported at present. We need to study whether these factors also affect the squatting ability of patients after TKA. Although some previous studies have analysed the influence of individual factors on the ROM of patients after TKA, no comprehensive analysis of multiple factors has been conducted. In our study, we will perform a comprehensive analysis after screening these factors.

To the authors' knowledge, there have been no direct studies in the literature on factors that affect squatting after
TKA in Asians. The aim of the present study was to analyse the influence of multiple factors on the squatting ability of patients after TKA, and to determine which factor significantly affected squatting ability.

\section{Patients and Methods}

We retrospectively reviewed a case series of 200 knees who underwent primary TKA by a senior surgeon from 2009 to 2018. The patients included in our study satisfied the following criteria: (1) their preoperative diagnosis was osteoarthritis, and (2) high flexion prosthesis (PFC Sigma PS150; DePuy, Warsaw, IN, USA) was used for TKAs. The patients were excluded based on the following criteria: (1) joint replacement other than knee replacement, (2) follow-up $<3$ years, and (3) incomplete data.

Radiological data included a weightbearing full length lower extremity antero-posterior radiograph performed with a centered patella showing the entire lower limb, a medio-lateral view performed strictly perpendicular to the superior face of tibial condyles, and an anterioposterior view performed with a strictly centred patella. The sex, age, weight, and height of each subject were noted. Other operative data on the use of the prosthesis size, and the thickness of the polyethylene insert used were collected from the patients' electronic records. The most 
recent follow-up data were used. The patients were placed under general or spinal-epidural combined anaesthesia, and an air tourniquet was applied in all cases. In all knees the same surgical technique was followed, using standard intramedullary alignment blocks, standard ligament-balancing techniques, an anterior femoral referencing system, and a constant tibial articular slope of $3^{\circ}$. The operation and follow-up assessment were performed by different surgeons.

We divided the patients into two groups according to the squatting position and knee flexion angle in the weight-bearing group (Group I - inability to squat group, Group II - ability to squat group). During patient squatting, the patient's knee flexion angle should be at least 135 degrees as the critical angle. In addition, the critical position was when the inguinal fold was in a straight horizontal line with the top of the knee during the patient's squatting, and the squat position was defined as the top of the thighs falls below the horizontal plane. Our criteria for determining whether patients could squat were based on studies of Olympic athletes. ${ }^{7}$ In the inability to squat group (Group I), the knee flexion angle or squat position did not exceed the critical value during squatting. In the ability to squat group (Group II), the knee flexion angle was greater than the critical angle and the squatting position was greater than the critical position. Specifically, in the Group II, not only did all patients have knee flexion angles greater than $135^{\circ}$, but they could all reach the squat position. Each patient received routine preoperative and postoperative clinical care. Evaluations included serial radiographs, squatting ability, and all components of the Knee Society Score (KSS) system, Western Ontario and McMaster Universities (WOMAC).

\section{Radiographic Assessment}

Preoperative radiographs served as a baseline, to which all postoperative radiographs were compared. There is no standard anatomical measuring system to correctly identify the joint line. According to Hofmann's method, ${ }^{11}$ the JL defined as a line tangent to the most distal points of the medial and lateral femoral condyles, and the JL position was defined as the distance from the adductor tubercle to the JL. The JL position was evaluated on preoperative and postoperative knee radiographs in this method, and the changes were calculated. The Insall-Salvati ratio (ISR) on lateral radiographs taken at $30^{\circ}$ of flexion was used to evaluate the patellar position and diagnosis of patella baja and pseudopatella baja. ISR $<0.8$ was defined as patella baja (normal ISR is $1 \pm 0.2$ ), ${ }^{12}$ whereas ISR $\geq 0.8$ was defined as pseudo-patella baja. ${ }^{13}$

Lateral X-rays of the knee were used to measure posterior condylar offset (PCO). The preoperative PCO was measured on the true lateral radiograph, as the tangential distance between the posterior cortex of the femoral diaphysis and the apex of the posterior femoral condyle. The postoperative PCO was measured as the tangential distance between the posterior cortex of the femoral diaphysis and the apex of the posterior femoral component postoperatively. Then, the preoperative and postoperative measurements were compared. A radiograph of the knee anteroposterior view was used to assess the ratio of the transverse diameter of the patient's femoral prosthesis and the width of the distal femur. The distal femoral width (FW) was described as the line joining the medial and lateral epicondyles at their most prominent points. The radiographic evaluation was independently performed by the two authors and repeated by the two authors at twoweek intervals.

\section{Statistical Analysis}

All patients were divided into two groups: inability to squat group (Group I) and ability to squat group (Group II). Baseline characteristics and squatting ability were compared between the two groups. Descriptive statistics are reported as the mean and standard deviation, as the mean and range, or as the number and percentage. Univariate and multivariate logistic regression analyses were performed to estimate the odds ratio for the correlation between baseline characteristics and ORR. Before proceeding to multivariate logistic regression analysis for the factors, multico-linearity was checked by doing colinearity diagnostic tests and the variables with "variance inflation factor" (VIF) below 10 were selected for final model (multivariate logistic regression) analysis to identify factors which had significant association with the dependent variable, and no multicollinearity was detected. A p value of $<0.05$ was considered statistically significant. Analysis was performed using SPSS Version 25.0 software (IBM, Armonk, NY, USA).

\section{Results}

The baseline information including demographic and clinical data and prosthesis, is clearly listed in Table 1, whereas radiological data are summarized in Table 2. The KSS scores and WOMAC scores of the ability to squat group (Group II) were improved compared with 
Table I Demographic, Prosthetic and Clinical Data of Two Groups

\begin{tabular}{|c|c|c|c|}
\hline Variable & Group I & Group II & $\mathbf{P}$ \\
\hline Age (year) & $64.9 \pm 7.7$ & $61.7 \pm 6.6$ & $>0.05$ \\
\hline Gender (M/F) & $9 / 91$ & $14 / 86$ & $>0.05$ \\
\hline BMI (kg/m2) & $27.3 \pm 4.2$ & $27 \pm 3.2$ & $>0.05$ \\
\hline Preoperative ROM & $82.9 \pm 12.6$ & $107 \pm 9.6$ & $<0.01$ \\
\hline \multicolumn{4}{|l|}{ Model of prosthesis } \\
\hline Femoral & $2.73 \pm 0.8$ & $2.97 \pm 0.8$ & $>0.05$ \\
\hline Tibia & $2.58 \pm 0.62$ & $2.77 \pm 0.67$ & $>0.05$ \\
\hline Liner Thickness & $11.12 \pm 1.55$ & $10.85 \pm 1.43$ & $>0.05$ \\
\hline Patella resurfacing & $66(66 \%)$ & 94 (47\%) & $>0.05$ \\
\hline \multicolumn{4}{|l|}{ Primary diagnosis } \\
\hline Osteoarthritis & $90(90 \%)$ & $91(91 \%)$ & \\
\hline Rheumatoid arthritis & $7(7 \%)$ & $9(9 \%)$ & \\
\hline Others & $3(3 \%)$ & 0 & \\
\hline \multicolumn{4}{|l|}{ KSS } \\
\hline Post-operation & $82.5 \pm 7.4$ & $90 \pm 5.7$ & \\
\hline \multicolumn{4}{|l|}{ WOMAC } \\
\hline Post-operation & $18 \pm 6.5$ & $12 \pm 5.8$ & \\
\hline
\end{tabular}

Abbreviations: BMI, body mass index; WOMAC, Western Ontario and McMaster Universities; KSS, American knee society knee score.

Table 2 Radiographic Results

\begin{tabular}{|l|c|c|c|}
\hline Variable & Group I & Group II & P \\
\hline Patella baja after TKA (ISR < 0.8) & $10(10 \%)$ & $13(6.5 \%)$ & $>0.05$ \\
\hline $\begin{array}{l}\text { Joint line elevation } \\
\text { Post-TKA }\end{array}$ & $3.24 \pm 1.07$ & $1.83 \pm 0.89$ & $<0.01$ \\
\hline $\begin{array}{l}\text { Changes in PCO } \\
\text { Post- TKA }\end{array}$ & $0.61 \pm 1.38$ & $0.47 \pm 1.10$ & $>0.05$ \\
\hline FC/FM & $0.88 \pm 0.09$ & $0.87 \pm 0.06$ & $>0.05$ \\
\hline
\end{tabular}

Abbreviations: ISR, Insall-Salvati ratio; PCO, posterior condylar offset; FW, the femoral width, described as the line joining the medial and lateral epicondyles at their most prominent points; FC, femoral side component width.

those of the inability to squat group (Group I), but the improvement in scores was not significant.

Univariate logistic regression analysis of baseline factors affecting ORR was performed for the following factors: age, sex, BMI, prosthesis size, patella resurfacing, patella baja, JL position, preoperative ROM, FC/FM, and changes in PCO. Age, prosthesis size, preoperative ROM and JL position showed $p$ value $<0.10$ in the univariate analyses. Variables with a $\mathrm{p}$ value $<0.1$ in univariate analyses were included in the multivariate analysis. $\mathrm{P}<0.05$ was considered significant in the multivariate analysis. The multivariate analysis identified JL position and preoperative ROM as independent predictors of ORR $(\mathrm{p}<0.01)$ (Table 3). JL position and preoperative ROM were independent influencing factors that affected squatting ability after TKA.

\section{Discussion}

The factors affecting squatting ability after TKA have not been reported in the literature. The main finding of this study was that JL elevation and preoperative ROM were independent influencing factors affecting squatting ability after TKA.

The majority of literatures has reported the factors affecting the range of motion after TKA in non-weight bearing conditions. However, no previous studies have reported the factors affecting the patient's squatting ability after TKA. Our study focused on the factors affecting squatting ability after TKA in weight bearing conditions. In Asian populations, activities such as cross-legged sitting, kneeling, and squatting are an important part of daily activities. ${ }^{4}$ Therefore, it is necessary and important to clarify the influencing factors of squatting.

Some studies reported that sex had no correlation with knee ROM postoperatively after TKA. ${ }^{14-16}$ Bellemans et al ${ }^{17}$ found that the use of standard implants could lead to mediolateral overhang in women, causing irritation and pain of the soft tissue capsular envelope because female knees are narrower than male knees. The results of this study show that the relationship between sex and clinical outcomes remains uncertain. In our study, sex was not a factor that influenced patients' squats. However, the majority of subjects in our study were women, which may have influenced our final results.

Sancheti et $\mathrm{al}^{18}$ found that age and patellar replacement did not affect the postoperative joint ROM, whereas BMI had a negative influence on the postoperative joint ROM. In our study, age was related to postoperative squatting in univariate analysis, but after multivariate analysis, age, BMI, and patella replacement proved not to be the influencing factors of postoperative squatting. The study of Razzaki et al ${ }^{19}$ specifically focused on about the relationship between BMI and clinical outcome in patients. They found that the Knee Society Knee Score was significantly higher in the patients who gained weight, whereas the patients who lost weight had the highest Oxford Knee Score and the lowest Knee Society Function Score. Additionally, weight change following TKA 
Table 3 Univariate and Multivariate Logistic Regression Analysis for Factors Affecting ORR

\begin{tabular}{|c|c|c|c|c|c|c|}
\hline \multirow[t]{2}{*}{ Variable } & \multirow[t]{2}{*}{ Group I } & \multirow[t]{2}{*}{ Group II } & \multicolumn{2}{|l|}{ Univariate Analysis } & \multicolumn{2}{|l|}{ Multivariate Analysis } \\
\hline & & & Odds Ratio $(95 \% \mathrm{Cl})$ & $\mathbf{p}$ & Odds Ratio (95\% Cl) & $\mathbf{p}$ \\
\hline Gender (M/F) & 9/91 & $14 / 86$ & $0.98(0.42-2.26)$ & $>0.1$ & $\longrightarrow$ & \\
\hline BMI (kg/m2) & $27.3 \pm 4.2$ & $27 \pm 3.2$ & $0.98(0.91-1.05)$ & $>0.1$ & - & \\
\hline Patella resurfacing & $66(66 \%)$ & 94 (47\%) & $1.46(0.80-2.66)$ & $>0.1$ & - & \\
\hline patella baja & $10(10 \%)$ & $13(6.5 \%)$ & $0.66(0.28-1.59)$ & $>0.1$ & - & \\
\hline Changes in $\mathrm{PCO}$ & $0.61 \pm 1.38$ & $0.47 \pm 1.10$ & $0.91(0.73-1.14)$ & $>0.1$ & - & \\
\hline $\mathrm{FC} / \mathrm{FM}$ & $0.88 \pm 0.09$ & $0.87 \pm 0.06$ & $0.10(0.00-4.08)$ & $>0.1$ & - & \\
\hline Age (year) & $64.9 \pm 7.7$ & $61.7 \pm 6.6$ & $0.94(0.90-0.98)$ & $<0.1$ & $0.97(0.88-1.07)$ & $>0.05$ \\
\hline joint line elevation & $3.24 \pm 1.07$ & $1.83 \pm 0.89$ & $0.22(0.15-0.34)$ & $<0.1$ & $0.09(0.32-0.25)$ & $<0.01$ \\
\hline \multicolumn{7}{|l|}{ Prosthesis size } \\
\hline Femoral & $2.73 \pm 0.8$ & $2.97 \pm 0.8$ & $1.57(1.07-2.30)$ & $<0.1$ & $1.04(0.20-5.54)$ & $>0.05$ \\
\hline Tibia & $2.58 \pm 0.62$ & $2.77 \pm 0.67$ & $1.56(0.99-2.44)$ & $<0.1$ & $2.98(0.43-20.76)$ & $>0.05$ \\
\hline Liner Thickness & $11.12 \pm 1.55$ & $10.85 \pm 1.43$ & $0.83(0.7 \mathrm{I}-0.98)$ & $<0.1$ & $0.95(0.66-1.37)$ & $>0.05$ \\
\hline Preoperative ROM & $82.9 \pm 12.6$ & $107 \pm 9.6$ & $1.23(1.16-1.30)$ & $<0.1$ & $1.34(1.20-1.49)$ & $<0.01$ \\
\hline
\end{tabular}

Abbreviations: BMI, body mass index; ISR, Insall-Salvati ratio; PCO, posterior condylar offset; FW, the femoral width, described as the line joining the medial and lateral epicondyles at their most prominent points; FC, femoral side component width; ROM, range of motion.

did not affect clinical outcomes, which remained good across all BMI groups.

Physiological patellofemoral joint motion was important for knee flexion function. ISR $<0.8$ was defined as patella baja (PB). PB is known to affect patellofemoral joint motion; a shortening of more than $10 \%$ of the tendon has been hypothesized to significantly reduce knee flexion due to shortening of the extensor mechanism length. ${ }^{10,20,21}$ There have also been many reports in the literature that patella baja can restrict the ROM of the knee. ${ }^{22-24}$ However, recent studies have shown that patella baja does not affect the patient's postoperative $\mathrm{ROM}^{25,26}$ In our study, patella baja $(\mathrm{P}>0.05)$ was also not an influencing factor of squatting. Bellemans et $\mathrm{al}^{27}$ defined a parameter termed "posterior condylar offset." They found that PCO was associated with a postoperative change in flexion angle in CR knees, whereas this correlation was not observed in PS knees. Yang et $\mathrm{al}^{28}$ found that PCO did not seem to have a significant influence on postoperative knee flexion after PS TKA. The prostheses of the patients in our study were all PS knees, which also explained why PCO had no effect on the postoperative squatting ability in our findings.

Oversizing the implant could theoretically increase tension and capsule/ligament friction on the implant, thus affecting clinical outcomes. ${ }^{29}$ Bonnin et $\mathrm{al}^{29}$ found that oversizing of the lateral side of the prosthesis was a factor that may predict poor TKA results. Therefore, we included the prosthesis size and FC/FM to determine whether these factors would affect the patient's squatting ability. The results showed neither the model of the prosthesis nor FC/FM can affect squatting ability after TKA. We believe that the reason for this result is that this surgeon has a superb surgical technique and can accurately select the appropriate prosthesis model for different patients, as it can be seen from our data that the FC/FM $(0.88 \pm 0.09$ vs $0.87 \pm 0.06)$ of Group I and Group II are similar, so we cannot be sure the model of the prosthesis and FC/FM did not affect the squatting ability.

Gatha et $\mathrm{al}^{30}$ assessed whether specific preoperative and postoperative variables were associated with postoperative ROM in 135 patients who underwent TKA. Their results showed that preoperative ROM was the only significant predictor of postoperative ROM. Ritter et $\mathrm{al}^{31}$ retrospectively reviewed more than 4700 TKA procedures and used regression tree analysis to describe the combination of variables affecting postoperative ROM. The primary predictor of postoperative ROM was preoperative ROM. In a recent study, Konrads et $\mathrm{al}^{32}$ demonstrated that preoperative ROM is a good predictor of postoperative ROM after TKA. The results of these studies were similar to those of our study, in which preoperative ROM was a contributing factor affecting the postoperative squatting ability.

Mean JL elevation 1.1-5.6 mm after primary TKA. ${ }^{33}$ Some studies have reported a correlation between elevated JLs and patients' postoperative $\mathrm{ROM},{ }^{25,34,35}$ whereas others have found no such correlation. ${ }^{36}$ There are currently many explanations for why elevated JLs lead to 
reduced ROM. One view was that elevation of the JL could lead to mid-flexion instability, which reduced the ROM after TKA. ${ }^{37}$ Matziolis et $\mathrm{al}^{38}$ also found JL position was associated with mid-flexion instability, which could lead to a reduced ROM. The term "mid-flexion instability" is a definition to describe instability during flexion-extension movement, which has not been proven to be a unique type of instability in clinical practice. ${ }^{39}$ However, another view is that $\mathrm{JL}$ elevation causing patella baja can result in reduced force of the extensor mechanism, patellar impingement on the insert caused anterior knee pain, and tightening of collateral ligaments and diminished femoral rollback resulted in limited knee motion. ${ }^{40}$ In our study, we performed univariate analysis with both patella baja and joint line elevation as influencing factors, and the results showed that patella baja was not a factor affecting ROM. Therefore, in view of our results, JL elevation led to mid-flexion instability, which affected the squatting ability of patients after TKA.

There were also limitations in this study. First, it has limited generalizability as our cohort belongs to a single surgeon and a single institution, and women made up the majority. Second, we only preliminarily defined squatting according to the flexion angle of the knee joint in weightbearing conditions because there is no formal definition of squatting in patients after TKA.

\section{Conclusion}

This study demonstrated that JL elevation and preoperative ROM were independent influencing factors affecting squatting ability after TKA. Patients with poor preoperative ROM should be counseled that they may not achieve satisfactory squatting ability postoperatively. In addition, to ensure that patients can squat after TKA, we should focus on controlling the JL elevation. The JL elevation was determined by the surgical technique, and whether the patient can squat after TKA is a test of the surgeon's skill.

\section{Abbreviations}

TKA, total knee arthroplasty; BMI, body mass index; WOMAC, Western Ontario and McMaster Universities; KSS, American knee society knee score; ISR, InsallSalvati ratio; PCO, posterior condylar offset; FW, the femoral width, described as the line joining the medial and lateral epicondyles at their most prominent points; FC, femoral side component width; ROM, range of motion.

\section{Data Sharing Statement}

All data generated or analyzed during this study are included in this published article.

\section{Ethics Approval and Informed Consent}

This retrospective review study involving human participants was in accordance with the ethical standards of the institutional and national research committee and with the 1964 Helsinki Declaration and its later amendments or comparable ethical standards. The Ethics Committee of our hospital, General Hospital of Chinese People's Liberation Army, approved the study protocol. All the study participants provided written informed consent for the study.

\section{Acknowledgments}

The authors would like to thank all staff from the participating departments and clinics.

\section{Author Contributions}

All authors made a significant contribution to the work reported, whether that is in the conception, study design, execution, acquisition of data, analysis and interpretation, or in all these areas; took part in drafting, revising or critically reviewing the article; gave final approval of the version to be published; have agreed on the journal to which the article has been submitted; and agree to be accountable for all aspects of the work.

\section{Funding}

This research did not receive any specific grant from funding agencies in the public, commercial, or not-forprofit sectors.

\section{Disclosure}

The authors declare that they have no competing interests.

\section{References}

1. Kahn TL, Soheili A, Schwarzkopf R. Outcomes of total knee arthroplasty in relation to preoperative patient-reported and radiographic measures: data from the osteoarthritis initiative. Geriatr Orthop Surg Rehabil. 2013;4(4):117-126. doi:10.1177/2151458514520634

2. Bourne RB, Chesworth BM, Davis AM, Mahomed NN, Charron KD. Patient satisfaction after total knee arthroplasty: who is satisfied and who is not? Clin Orthop Relat Res. 2010;468(1):57-63. doi:10.1007/ s11999-009-1119-9 
3. Clement ND, MacDonald D, Patton JT, Burnett R. Post-operative Oxford knee score can be used to indicate whether patient expectations have been achieved after primary total knee arthroplasty. Knee Surg Sports Traumatol Arthrosc. 2015;23(6):1578-1590. doi:10.1007/ s00167-014-2865-0

4. Park KK, Chang CB, Kang YG, Seong SC, Kim TK. Correlation of maximum flexion with clinical outcome after total knee replacement in Asian patients. J Bone Joint Surg Br. 2007;89(5):604-608. doi:10.1302/0301-620X.89B5.18117

5. Laubenthal KN, Smidt GL, Kettelkamp DB. A quantitative analysis of knee motion during activities of daily living. Phys Ther. 1972;52 (1):34-43. doi:10.1093/ptj/52.1.34

6. Ritter MA, Campbell ED, Ritter MA, Campbell ED. Effect of range of motion on the success of a total knee arthroplasty. J Arthroplasty. 1987;2(2):95-97. doi:10.1016/S0883-5403(87)80015-3

7. Hartmann H, Wirth K, Klusemann M. Analysis of the load on the knee joint and vertebral column with changes in squatting depth and weight load. Sports Med. 2013;43(10):993-1008. doi:10.1007/ s40279-013-0073-6

8. Kawamura H, Bourne RB. Factors affecting range of flexion after total knee arthroplasty. $J$ Orthop Sci. 2001;6(3):248-252. doi:10.1007/s007760100043

9. Malviya A, Lingard EA, Weir DJ, Deehan DJ. Predicting range of movement after knee replacement: the importance of posterior condylar offset and tibial slope. Knee Surg Sports Traumatol Arthrosc. 2009;17(5):491-498. doi:10.1007/s00167-008-0712-x

10. Babazadeh S, Dowsey MM, Swan JD, Stoney JD, Choong PF. Joint line position correlates with function after primary total knee replacement: a randomised controlled trial comparing conventional and computer-assisted surgery. $J$ Bone Joint Surg Br. 2011;93 (9):1223-1231. doi:10.1302/0301-620X.93B9.26950

11. Hofmann AA, Kurtin SM, Lyons S, Tanner AM, Bolognesi MP. Clinical and radiographic analysis of accurate restoration of the joint line in revision total knee arthroplasty. $J$ Arthroplasty. 2006;21 (8):1154-1162. doi:10.1016/j.arth.2005.10.026

12. Chonko DJ, Lombardi AV Jr, Berend KR. Patella baja and total knee arthroplasty (TKA): etiology, diagnosis, and management. Surg Technol Int. 2004;12:231-238.

13. Grelsamer RP. Patella baja after total knee arthroplasty: is it really patella baja? J Arthroplasty. 2002;17(1):66-69. doi:10.1054/ arth.2002.28728

14. Insall J, Scott WN, Ranawat CS. The total condylar knee prosthesis. A report of two hundred and twenty cases. J Bone Joint Surg Am. 1979;61(2):173-180. doi:10.2106/00004623-197961020-00003

15. Kotani A, Yonekura A, Bourne RB. Factors influencing range of motion after contemporary total knee arthroplasty. $J$ Arthroplasty. 2005;20(7):850-856. doi:10.1016/j.arth.2004.12.051

16. Ishii Y, Matsuda Y, Sakata S, Onda N, Omori G. Primary total knee arthroplasty using the Genesis I total knee prosthesis: a 5- to 10-year follow-up study. Knee. 2005;12(5):341-345. doi:10.1016/j. knee.2004.12.004

17. Bellemans J, Carpentier K, Vandenneucker H, Vanlauwe J, Victor J. The John Insall Award: both morphotype and gender influence the shape of the knee in patients undergoing TKA. Clin Orthop Relat Res. 2010;468(1):29-36. doi:10.1007/s11999-009-1016-2

18. Sancheti KH, Sancheti PK, Shyam AK, Joshi R, Patil K, Jain A. Factors affecting range of motion in total knee arthroplasty using high flexion prosthesis: a prospective study. Indian J Orthop. 2013;47 (1):50-56. doi:10.4103/0019-5413.106901

19. Razzaki T, Mak WK, Bin Abd Razak HR, Tan HA. Patterns of weight change and their effects on clinical outcomes following total knee arthroplasty in an Asian Population. J Arthroplasty. 2020;35 (2):375-379. doi:10.1016/j.arth.2019.09.003

20. Bellemans J. Restoring the joint line in revision TKA: does it matter? Knee. 2004;11(1):3-5. doi:10.1016/S0968-0160(03)00099-1
21. Behrend H, Graulich T, Gerlach R, Spross C, Ladurner A. Blackburne-Peel ratio predicts patients' outcomes after total knee arthroplasty. Knee Surg Sports Traumatol Arthrosc. 2019;27 (5):1562-1569.

22. Caton J, Deschamps G, Chambat P, Lerat JL, Dejour H. [Patella infera. Apropos of 128 cases]. Rev Chir Orthop Reparatrice Appar Mot. 1982;68(5):317-325. Swedish.

23. Weale AE, Murray DW, Newman JH, Ackroyd CE. The length of the patellar tendon after unicompartmental and total knee replacement. J Bone Joint Surg Br. 1999;81(5):790-795. doi:10.1302/0301620X.81B5.0810790

24. Bruhin VF, Preiss S, Salzmann GM, Harder LP. Frontal tendon lengthening plasty for treatment of structural patella baja. Arthrosc Tech. 2016;5(6):e1395-e1400. doi:10.1016/j.eats.2016.08.004

25. Han HS, Yu CH, Shin N, Won S, Lee MC. Femoral joint line restoration is a major determinant of postoperative range of motion in revision total knee arthroplasty. Knee Surg Sports Traumatol Arthrosc. 2019;27(7):2090-2095. doi:10.1007/s00167-019-05361-1

26. Graulich T, Kranz C, Zhang D, et al. Reduction of patella-baja and pseudo-patella-baja does not improve range of motion in patients after mega-TKA. Vivo. 2020;34(3):1153-1158. doi:10.21873/ invivo. 11887

27. Bellemans J, Banks S, Victor J, Vandenneucker H, Moemans A. Fluoroscopic analysis of the kinematics of deep flexion in total knee arthroplasty. Influence of posterior condylar offset. J Bone Joint Surg Br. 2002;84(1):50-53. doi:10.1302/0301-620X.84B1.0840050

28. Yang G, Chen W, Chen W, et al. Full-thickness cartilage-based posterior femoral condylar offset. Influence on knee flexion after posterior-stabilized total knee arthroplasty. Orthop Traumatol Surg Res. 2016;102(4):441-446. doi:10.1016/j.otsr.2016.02.011

29. Bonnin MP, Schmidt A, Basiglini L, Bossard N, Dantony E. Mediolateral oversizing influences pain, function, and flexion after TKA. Knee Surg Sports Traumatol Arthrosc. 2013;21 (10):2314-2324. doi:10.1007/s00167-013-2443-x

30. Gatha NM, Clarke HD, Fuchs R, Scuderi GR, Insall JN. Factors affecting postoperative range of motion after total knee arthroplasty. J Knee Surg. 2004;17(4):196-202. doi:10.1055/s-0030-1248221

31. Ritter MA, Harty LD, Davis KE, Meding JB, Berend ME. Predicting range of motion after total knee arthroplasty. Clustering, log-linear regression, and regression tree analysis. J Bone Joint Surg Am. 2003;85(7):1278-1285. doi:10.2106/00004623-200307000-00014

32. Konrads C, Rejaibia J, Grosse LC, et al. Patella-height analysis and correlation with clinical outcome after primary total knee arthroplasty. J Orthop. 2021;23:169-174. doi:10.1016/j.jor.2021.01.001

33. Snider MG, Macdonald SJ. The influence of the posterior cruciate ligament and component design on joint line position after primary total knee arthroplasty. J Arthroplasty. 2009;24(7):1093-1098. doi:10.1016/j.arth.2008.08.009

34. Clavé A, Le Henaff G, Roger T, Maisongrosse P, Mabit C, Dubrana F. Joint line level in revision total knee replacement: assessment and functional results with an average of seven years follow-up. Int Orthop. 2016;40(8):1655-1662. doi:10.1007/s00264-015-3096-9

35. Ji SJ, Zhou YX, Jiang X, et al. Effect of joint line elevation after posterior-stabilized and cruciate-retaining total knee arthroplasty on clinical function and kinematics. Chin Med J. 2015;128 (21):2866-2872. doi:10.4103/0366-6999.168043

36. Yang JH, Seo JG, Moon YW, Kim MH. Joint line changes after navigation-assisted mobile-bearing TKA. Orthopedics. 2009;32(10 Suppl):35-39. doi:10.3928/01477447-20090915-57

37. Martin JW, Whiteside LA. The influence of joint line position on knee stability after condylar knee arthroplasty. Clin Orthop Relat Res. 1990;259:146-156.

38. Matziolis G, Brodt S, Windisch C, Roehner E. Changes of posterior condylar offset results in midflexion instability in single-radius total knee arthroplasty. Arch Orthop Trauma Surg. 2017;137(5):713-717. doi:10.1007/s00402-017-2671-5 
39. Longo UG, Candela V, Pirato F, Hirschmann MT, Becker R, Denaro V. Midflexion instability in total knee arthroplasty: a systematic review. Knee Surg Sports Traumatol Arthrosc. 2021;29 (2):370-380. doi:10.1007/s00167-020-05909-6
40. Kazemi SM, Daftari Besheli L, Eajazi A, et al. Pseudo-patella baja after total knee arthroplasty. Med Sci Monit. 2011;17(5):Cr292-296. doi:10.12659/MSM.881770

\section{Publish your work in this journal}

Therapeutics and Clinical Risk Management is an international, peerreviewed journal of clinical therapeutics and risk management, focusing on concise rapid reporting of clinical studies in all therapeutic areas, outcomes, safety, and programs for the effective, safe, and sustained use of medicines. This journal is indexed on PubMed Central, CAS,
EMBase, Scopus and the Elsevier Bibliographic databases. The manuscript management system is completely online and includes a very quick and fair peer-review system, which is all easy to use. Visit http://www.dovepress.com/testimonials.php to read real quotes from published authors. 